\title{
Endoscopic Transnasal Interseptal Approach for Invasive Clival Tumors: Development of an Approach Method Regarding Maximal Preservation of the Nasal Anatomy
}

\author{
Masahiro SHIn, ${ }^{1}$ Kenji Kondo, ${ }^{2}$ Taichi KIn, ${ }^{1}$ Keigo SuzuKawA, ${ }^{2}$ \\ and Nobuhito SAITO ${ }^{1}$ \\ Departments of ${ }^{1}$ Neurosurgery and ${ }^{2}$ Otolaryngology, The University of Tokyo Hospital, Tokyo
}

\begin{abstract}
Asian people frequently show small noses, narrow nasal apertures, and congestive mucosa on the turbinates and septum. To reduce the risk of nasal morbidity with increased radicality for skull base tumors in these patients, we developed endoscopic transnasal interseptal approach (ETISA) in transsphenoidal surgery for invasive clival tumors (ICTs). Indication for ETISA is radical resection for tumors occupying deep ventral skull base regions, confined posterior to the level of the middle turbinates. After removing ethmoidal air cells, the middle turbinates are laterally deflected. A linear incision is made vertically on each side of the septal mucosa, which is separated from the bony septum as far as the sphenoid rostrum. The blades of an adjustable speculum are inserted submucosally, and the bony septum is temporarily displaced. The surgical pathway is widely maintained from the entrance to the deep surgical field without sacrificing the nasal mucosa and turbinates. Thirty-two consecutive patients with clival tumors (18 chordomas, 11 chondrosarcomas, 3 others) were treated. Bilateral middle turbinectomy was routinely performed in the initial 3 patients, but in only 4 of the remaining 29 ( 3 unilaterally, 1 bilaterally). Tumor was sufficiently resected in 29 patients $(90.6 \%$; gross total removal $n=25$, subtotal resection $n=4)$. As complications, 6 patients showed mild and transient worsening of cranial nerve symptoms. Nasal complications arose in 3 patients, persisting $>3$ months in $2(5.7 \%)$. This approach allows fine bimanual handling and swift delivery of surgical equipment while reasonably preserving the nasal anatomy, which is useful in endoscopic transsphenoidal surgery for ICT.
\end{abstract}

Key words: endoscopic transsphenoidal surgery, clival tumor, chordoma, chondrosarcoma, craniovertebral junction

\section{Introduction}

In the last decade, progress in endoscopic technology and techniques have dramatically changed the role of transsphenoidal surgery in the neurosurgical field. ${ }^{1)}$ Not only pituitary and parasellar lesions, but also various types of invasive skull base tumors can now be safely approached through the nostrils under direct vision. ${ }^{2-9)}$

In Asian countries including Japan, endoscopic surgery has been introduced over the last decade and has largely replaced conventional sublabial transsphenoidal surgery. ${ }^{10-12)}$ However, Asian individuals usually show a smaller nasal route than those of an European (white) or an African (black) background,

Received August 7, 2014; Accepted October 28, 2014 mainly resulting from not only the narrow nasal apertures, ${ }^{13)}$ but also the smaller volume of the nasal cavity with an increased proportion of vascular tissue in nasal mucosa. ${ }^{14-16)}$ To stably maintain a wide and clear surgical pathway to deep skull base regions, extensive dissection of the septal mucosa and middle turbinectomy have become essential in many cases. ${ }^{10,17-19)}$ Damage to the normal anatomy may become more extensive, raising concerns about the risk of nasal morbidity which continues even after the denuded mucosal surface has been healed by mucosalization. ${ }^{20-26)}$

From 1998, we started applying a single-nostril transseptal approach for pituitary tumors using a neuroendoscope in transsphenoidal surgery, and with the increase in surgical treatment of complex lesions, we started performing expanded endonasal 
surgeries for skull base tumors. ${ }^{11)}$ Through these experiences, we have gained an appreciation of the difficulties involved in balancing the radicality of surgery with the risk of nasal morbidity in deep skull base lesions in the patients with congestive nasal mucosa. This urged us to develop an approach method to minimize postoperative nasal complications while simultaneously improving the operability of surgical equipment for these procedures. Herein, we present our surgical technique for endoscopic transnasal interseptal approach (ETISA) in transsphenoidal surgery and the preliminary outcomes for 32 invasive clival tumors.

\section{Patients and Methods}

From 2009 to 2013, we have performed endoscopic transnasal surgery for 72 skull base tumors (excluding pituitary tumors and tumors limited in the suprasellar regions). Among these, 32 clival tumors were treated with 37 procedures using the ETISA (Fig. 1). The indication for this approach is the need for radical resection of tumors occupying the deep ventral skull base regions, confined posterior to the level of the middle turbinates. Median patient age was 55 years (range, 15-77 years). Other than this approach, we adopted a direct endonasal approach (31 tumors) for tumors invading superficial regions, such as the anterior cranial base or nasal cavity, tumors mainly located in the lateral regions such as periorbital region or the pterygopalatine fossa, and for tumors in the pediatric patients. When only biopsy was intended, a single-nostril transseptal approach was adopted (nine tumors).

The 32 clival tumors treated using ETISA comprised 18 chordomas, 11 chondrosarocomas, and 3 others (vascular tumor in clivus, clear cell meningioma, and giant cell tumor, 1 each). In them, 5 (15.6\%) referred to us after failure of multiple interventions including radical resections and various types of radiotherapy (heavy-particle radiotherapy, cyber knife radiosurgery, gamma knife radiosurgery). The tumors showed invasion to cavernous sinus in $24(75.0 \%)$, petrous region in $15(46.9 \%)$, jugular foramen or tubercle in $13(40.6 \%)$, and pharyngeal region in $9(28.1 \%)$. Intradural invasion was also observed in 5 tumors (15.6\%). In 30 patients $(93.8 \%)$, the tumors involved lateral regions such as the cavernous sinus, petrous region, middle cranial fossa, or jugular foramen.

\section{Surgical equipment specific for endoscopic surgery}

The surgical system for endoscopic surgery consists of a high-vision endoscope (rigid scopes of 175-180 mm in length), camera with cable, fiberoptic cable, light-emitting diode light source, and the monitor (Karl Storz Endoscopy Japan, Tokyo). Endoscopes with 4-mm diameter and $0^{\circ}$ or $30^{\circ}$ lenses are mainly used, while $70^{\circ}$ lenses are used on occasion. A high-frequency radiosurgical device with sharp malleable tip (Surgitron ${ }^{\circledR}$; Ellman International, New York, USA) is used to incise the nasal mucosa. The endoscope is stabilized in the surgical field with a robotic holding device (Point Setter; Mitaka Kohki, Tokyo). A neuronavigation system (StealthStation Navigation; Medtronic Japan, Tokyo) is routinely equipped.

A suction tube with irrigating function is essential to clear the endoscopic lens when clouded by mucus or blood (Fujita Medical Instruments Co., Ltd., Tokyo). Narrow-shaft bipolar cauterization and various dissecting or curetting devices are used simultaneously. To allow instruments to reach the lateral regions through the sphenoid sinus, the tips of all the instruments (ring-curette, scissors, suction with irrigating function, and bipolar cautery forceps)

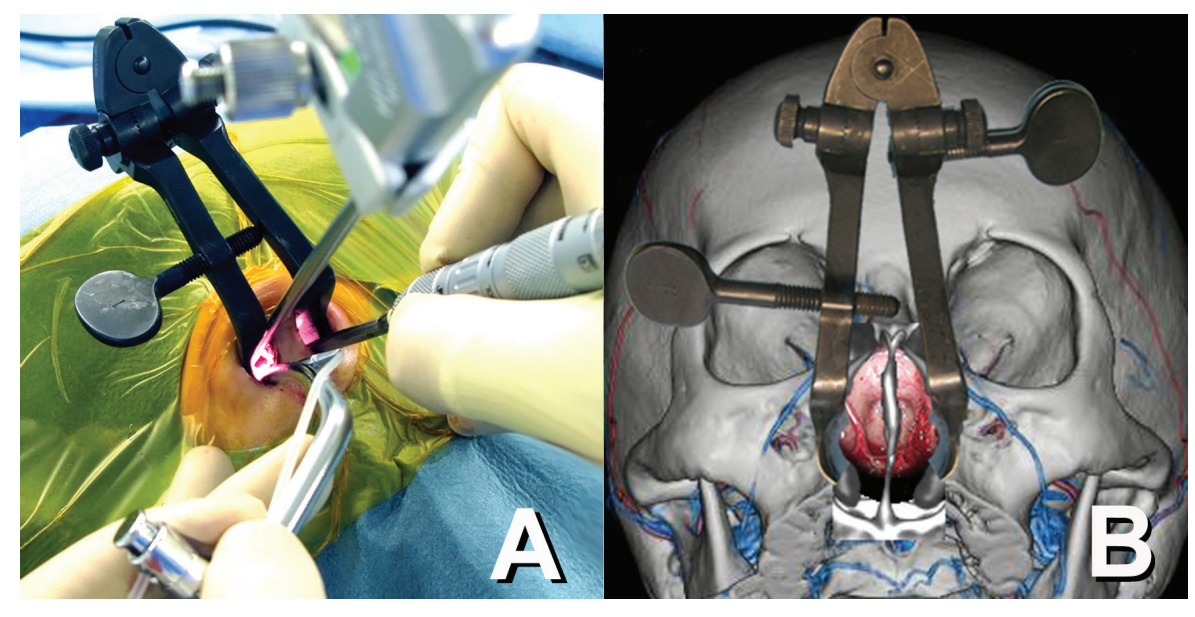

Fig. 1 Overview and basic positioning of surgical instruments in the binostril interseptal approach. A: The endoscope is introduced into the operative field from the rostral side to facilitate smooth bimanual surgical procedures. B: Schematic drawing. 
are designed to be curved in various degrees or to be malleable.

\section{Surgical procedure}

The patient is placed in a supine position with the head raised by $15^{\circ}$ and fixed with a Mayfield 3-point-head holder, rotated slightly toward the operator's side. After both nasal cavities are sterilized, nasal pledgets soaked in $0.02 \%$ epinephrine solution are placed on the nasal mucosa, which are removed at the last minute before starting surgery. Intraoperative monitoring of extraocular muscle movement was performed using electromyography to avoid the postoperative cranial nerve deficit.

To facilitate the surgical approach while maximally preserving the middle turbinates, the ethmoidal air cells are removed, and the uncinate process is also deflected laterally as necessary (Fig. 2A-C). The middle nasal turbinates are dislocated and thrust into the medial wall of the maxillary sinus to retain a sufficient approach route inside.

The mucosa on the nasal septum is incised in the rostro-caudal direction in linear fashion bilaterally at different depths of the nasal cavity on each side to avoid postoperative septal perforation; at the depth of $3 \mathrm{~cm}$ from the nasal entrance on the septal cartilage in one side, and at the level of the middle nasal turbinate on the bony septum in the other (Fig. 2D-F). The mucosa is separated from the septum and anterior aspects of the sphenoid bone (Fig. 2G-I). The mucosal retractors are inserted submucosally to push the dissected mucosae to

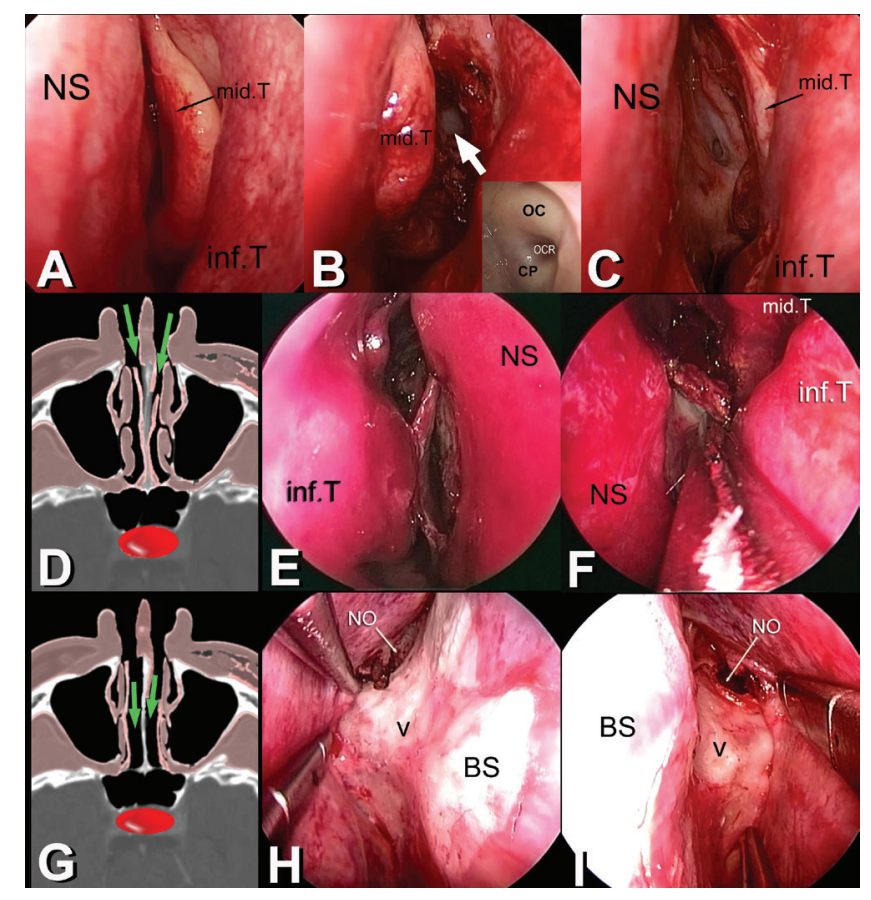

the sides, then the blades of the adjustable nasal speculum, specially designed to change the opening angle of the blades according to the shape and width of the nasal cavity (Fujita Medical Instruments), ${ }^{27)}$ are inserted into each nasal cavity (Figs. 1B, 3A-C). The bony septum is temporarily removed posterior to the mucosal incision to leave a marginal attachment of the mucosa for reconstruction at the end of the surgery. Because the blades of the adjustable speculum are detachable, to facilitate binostril submucosal insertion of the specular blades, they are inserted separately on each side and assembled after optimal submucosal insertion into each side (Fig. 3A-C). The speculum blades push the nasal mucosa laterally and gain maximum exposure of the anterior aspects of the sphenoid sinus or ventral skull base areas (Fig. 3D). The mucosae on the sphenoid rostrum are preserved with vascular supply from the septal branches of the sphenopalatine artery. The bone of the sphenoid rostrum is removed and the opening of the sphenoid sinus is enlarged.

In the tumor extending into the sphenoid sinus, the tumor is carefully dissected from its margin and is resected piece by piece. In deep lateral regions, we can swiftly transfer the variously curved surgical instruments along the nasal specula, without getting stuck in the nasal cavity, facilitating surgical procedures under the angled lens. When the tumor is located in the lower clivus or extends into the upper pharyngeal area, the bottom edge of the septal mucosa is incised and the septal mucosa is separated bilaterally. The blades of the nasal

Fig. 2 A-C: Observation of the left nasal cavity with a $0^{\circ}$ endoscope. In the left nasal cavity inspected with a $0^{\circ}$ rigid endoscope, the middle turbinate occupies a large portion of the space. The ethmoid air cells (EACs) are removed (B), and the superolateral margin of the sphenoid sinus is observed through the cavity (white arrow in B, close view in the lower right corner). After sufficient space is made lateral to the middle turbinate, the uncinate process is deflected, facilitating lateral dislocation of the middle nasal turbinates $(\mathrm{C})$ and retaining a sufficient approach space without turbinectomy. D, G: Schematic representation of the axial section of the nasal cavities. E, H: Observation of the right nasal cavity with a $0^{\circ}$ endoscope. F, I: Observation of the left nasal cavity. The mucosa on the nasal septum is incised perpendicularly in linear fashion at different depths to avoid postoperative septal perforation (D-F). The mucosa is separated from the bony septum (BS) and anterior aspects of the sphenoid bone (V) on each side, and the natural ostia (NO) are observed ( $\mathrm{H}$ and I). CP: carotid prominence, inf.T: inferior turbinate, mid.T: middle turbinate, NS: nasal septum. OC: optic canal, OCR: optico-carotid recess. 


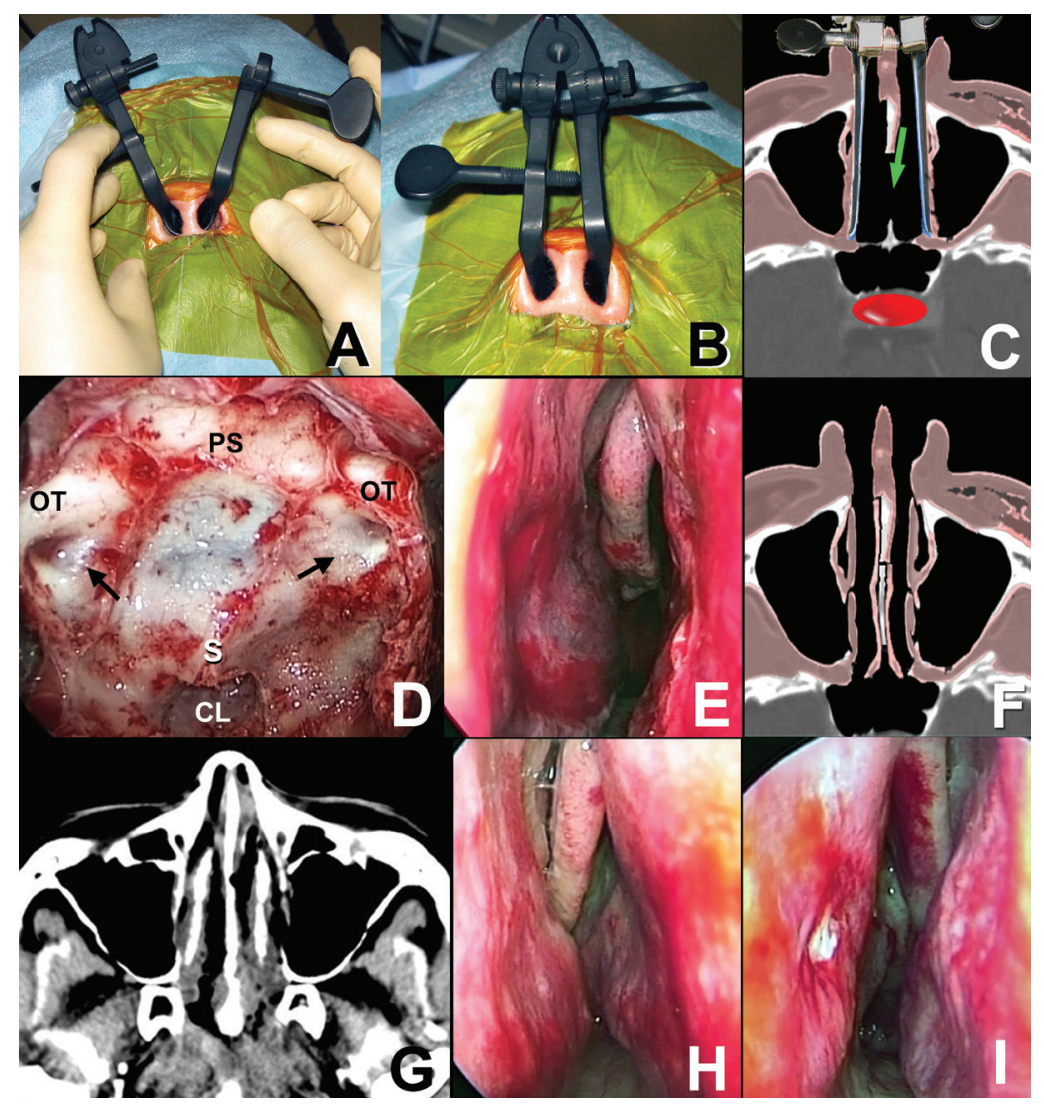

Fig. 3 A: The blades of the speculum are detached and inserted separately on each side. B: The blades are then assembled after optimal submucosal insertion into each side. C, D: Speculum blades push the nasal mucosa laterally (C: schematic drawing) and gain maximum exposure of the anterior aspects of the sphenoid sinus and ventral skull base areas, including bilateral optic canals (OCs), carotid prominences (arrows), planum sphenoidale (PS), sellar floor (S), and clivus (CL) (D). At the end of surgery, bony septa are replaced, and the septal mucosae and nasal turbinates are returned to their original positions. E: Observation of the right nasal cavity with a $0^{\circ}$ endoscope. F: Schematic representation of the axial section of the nasal cavities. G: Axial computed tomography immediately after surgical resection of the clival tumor. Inside and outside the middle turbinates, chitin-coated gauzes are packed in, and mucus and clot are also observed in the nasal cavity. H, I: One month after the surgery, endoscopic observation of the nasal cavity revealed healing of the septal incision with preservation of the nasal anatomy in the right $(\mathrm{H})$ and the left (I) nasal cavity. OT: optic tract. speculum are slid down and the upper pharynx is exposed with a sufficient approach pathway, without any sacrifice of the septal mucosa.

After tumor resection, in patients showing no cerebrospinal fluid (CSF) leakage during surgery, Gelfoam (Pfizer, Tokyo, Japan) with fibrin glue is placed in the cavity. When CSF leakage occurs from a small laceration of the sellar diaphragm or dura mater, abdominal adipose tissue is harvested and attached to the defect in the membrane using fibrin glue, then compressed by a sinus balloon inserted through the enlarged natural ostium of the sphenoid sinus. If the defect is relatively large and active CSF flow is observed continuously, the fascia lata is harvested, placed to cover the dural defect inside and outside the fistula and fixed with fibrin glue, then compressed by a sinus balloon. At the end of surgery, hemostasis and repair of CSF leakage are confirmed with the patient in a head-down position to raise the thoracic pressure, and tumor resection is completed. In cases where refractory CSF leakage is strongly anticipated, lumbar drainage is performed for 3-5 days. Use of a pedicled nasoseptal flap is considered at the last step of the surgery in limited cases.

After reconstruction of the skull base floor, the bony septum and mucosae are replaced and the posi- tions of the nasal turbinates are rectified (Fig. 3E, F), and Beschitin-F (chitin-coated gauze; Unitika, Co., Ltd., Okazaki, Aichi) is placed inside (between the nasal septum and middle turbinate) and outside the middle turbinates (between the middle turbinate and lateral wall of the nasal cavity) bilaterally for 36 hours to prevent synechia (Fig. 3G).

One week postoperatively, the patient undergoes postoperative endoscopic evaluation of the nasal cavities by an otolaryngologist. Nasal saline irrigation is continued at home for 1 month after discharge. Patients visit our outpatient clinic at 1 month and 3 months postoperatively for neurological evaluation and the second endoscopic rhinological check-ups (Fig. 3H, I).

\section{Results}

Surgical results are summarized in Table 1. In the initial 3 patients, the lower halves of bilateral middle turbinates were routinely resected. However, the necessity of this procedure was subsequently revised, and this procedure is now performed only in selected cases where sufficient surgical space cannot otherwise be achieved during surgery. In the most recent 29 patients, the septum and turbinates were completely preserved in 25 patients $(86.2 \%$, Fig. 4), and middle turbinectomy was necessary 
Table 1 Outcomes of 32 invasive clival tumors treated with endoscopic transnasal interseptal approaches

\begin{tabular}{|c|c|c|c|c|c|}
\hline Pathology & $\begin{array}{l}\text { Primary/ } \\
\text { Recurrent }\end{array}$ & $\mathrm{n}$ & $\begin{array}{l}\text { Previous } \\
\text { intervention }\end{array}$ & $\begin{array}{l}\text { Gross total } \\
\text { resection (rate) }\end{array}$ & Neurological complications \\
\hline \multirow[t]{3}{*}{ Chordoma } & Primary & 11 & - & $10(90.9 \%)$ & 0 \\
\hline & Recurrent & 7 & $\begin{array}{l}\text { CrS 2, TSS } 4 \text {, } \\
\text { RS } 2, \text { RT } 3\end{array}$ & $4(57.1 \%)$ & $\begin{array}{l}4 \text { (transient amnesia with DI 1, transient } \\
\text { CN palsy 3) }\end{array}$ \\
\hline & Total & 18 & & $14(77.8 \%)$ & \\
\hline \multirow[t]{3}{*}{ Chondrosarcoma } & Primary & 6 & - & $5(83.3 \%)$ & 2 (transient CN palsy 1, CSF rhinorrhea 1$)$ \\
\hline & Recurrent & 5 & $\mathrm{CrS} 5$ & $4(80.0 \%)$ & 1 (transient CN palsy 1) \\
\hline & Total & 11 & & $9(81.8 \%)$ & \\
\hline \multirow[t]{2}{*}{ Others $^{+}$} & Primary & 2 & - & $2(100 \%)$ & 1 (transient CN palsy 1) \\
\hline & Recurrent & 1 & CrS 1 , RS 1 & $0(0 \%)$ & 0 \\
\hline \multirow[t]{3}{*}{ All } & Primary & 19 & & $17(89.4 \%)$ & 3 \\
\hline & Recurrent & 13 & & $8(61.5 \%)$ & 5 \\
\hline & Total & 32 & & $25(78.1 \%)$ & 8 (transient CN palsy 6, others 2 ) \\
\hline
\end{tabular}

+ : involving angiomatosis, giant cell tumor, and clear cell meningioma (recurrent, World Health Organization grade II), CN: cranial nerve, CrS: transcranial surgery, CSF: cerebrospinal fluid, DI: transient diabetes insipidus, RS: radiosurgery, RT: fractionated radiotherapy, TSS: transsphenoidal surgery.

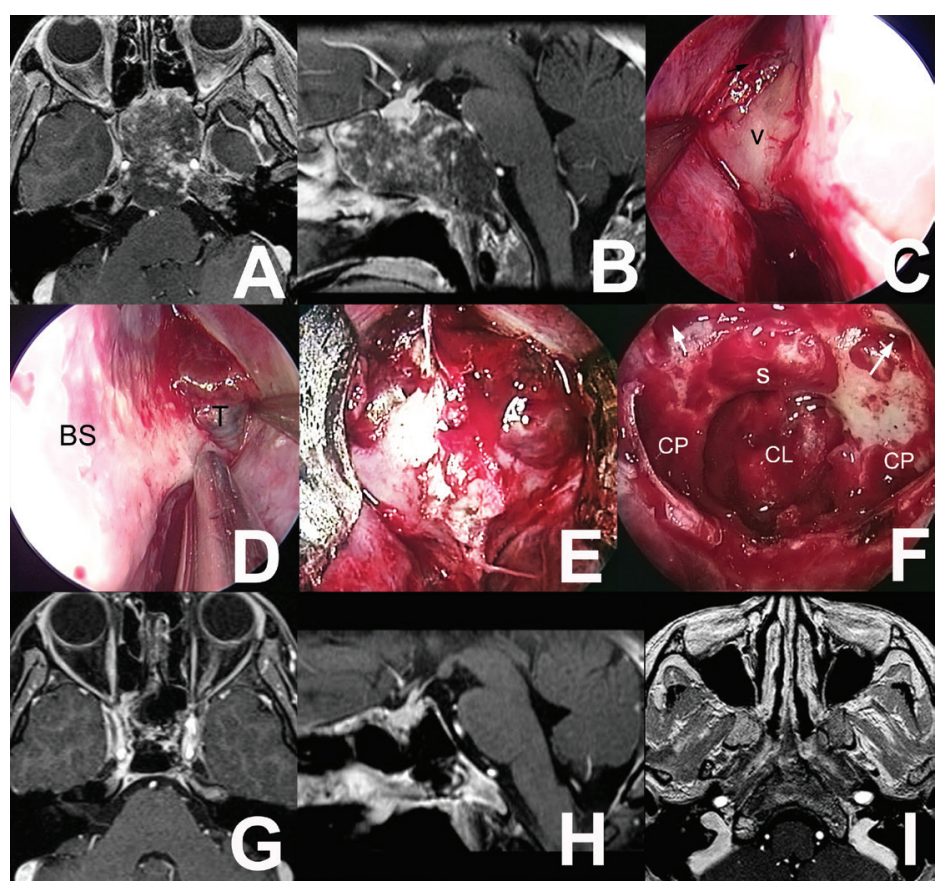

Fig. 4 A case of typical clival chordoma occupying the clivus and sphenoid sinus $\left(\mathrm{T}_{1}\right.$-weighted magnetic resonance imaging (MRI) with gadolinium enhancement; A: axial section, B: sagittal section). The patient presented with bilateral abducens nerve palsy and underwent ETISA using adjustable nasal specula. The mucosae on the nasal septum were incised perpendicularly in linear fashion and are separated from the bony septum (BS) (C: observation of right nasal cavity with a $0^{\circ}$ endoscope, D: observation of the left nasal cavity). The anterior aspect of the sphenoid bone was widely exposed (E). After gross total removal of the tumor, the normal anatomical structures including the sellar floor (S), bilateral optico-carotid recesses (arrows), carotid prominence (CP), and clivus (CL), are visualized $(F)$. Postoperative $T_{1}$-weighted MRI with gadolinium enhancement (G: axial section, $\mathrm{H}$ : coronal section) discloses successful removal of the tumor and complete preservation of the nasal mucosae and turbinates (I: axial section). The patient did not show any nasal complications, and abducens nerve palsies completely resolved within 6 months. ETISA: endoscopic transnasal interseptal approach, inf.T: inferior turbinate, mid.T: middle turbinate, T: tumor, V: anterior aspects of sphenoid bone. in 4 patients (13.8\%, Fig. 5), unilaterally in 3 and bilaterally with the lower one-third in 1 patient. Among these cases, the posterior tip of the inferior turbinate on the affected side was also removed in one patient because of tumor extension into the pterygopalatine fossa, and a lateral approach would have been difficult without this action.
In the 32 clival tumors, gross total removal was achieved in 25 (78.1\%). Of these, 19 tumors were treated primarily with the ETISA in our institution, achieving gross total resection of 17 tumors $(89.4 \%$ of primary cases). In the 30 tumors involving lateral regions, 26 were successfully removed through the lateral aspects of the sphenoid sinus under the 


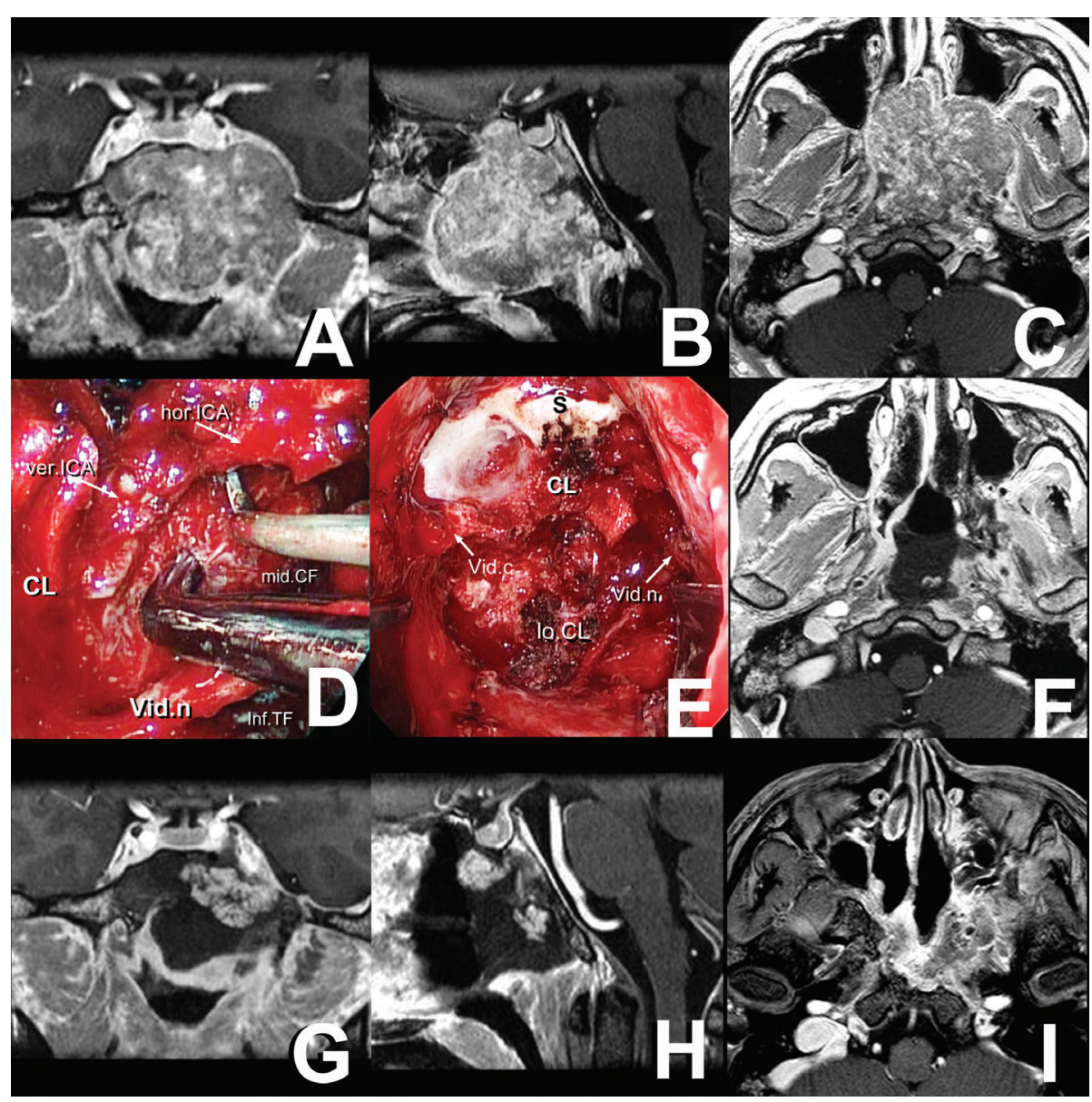

Fig. 5 A case of chordoma with extensive invasion into the sphenoid sinus, clivus, pterygopalatine fossa, middle cranial fossa, and infratemporal fossa. A-C: $\mathrm{T}_{1}$-weighted magnetic resonance imaging (MRI) with gadolinium enhancement (A: axial section, B: coronal section, C: sagittal section). Because the tumor showed marked extension behind the orifice of the left middle turbinate, we resected the lower half of the left middle turbinate, facilitating the approach to this region and also allowing direct approaches to the tumor in the pterygopalatine and infratemporal fossas. The tumor was dissected from the clivus (CL), and carefully from the internal carotid arteries. D: After removal of the midline component, the tumor extending into the middle cranial fossa (mid.CF), and infratemporal fossa (inf.TF) was resected under a $30^{\circ}$ scope through the surgical spaces above and below the left vidian nerve (Vid.n). E: After gross total removal of the tumor, normal anatomical structures including the sellar floor (S), upper and lower clivus (CL and lo.CL, respectively), right vidian canal (Vid.c), and left vidian nerve (Vid.n) were visualized. F-H: Postoperative $T_{1}$-weighted MRI with gadolinium enhancement (F: axial section, G: coronal section, H: sagittal section) disclosed the complete tumor removal. Postoperative MRI obtained 3 months after surgery revealed preservation of the residual nasal anatomies (I). The patient did not show any neurological complications after surgery. hor.ICA: horizontal portion of the internal carotid artery, ver. ICA: vertical portion of the internal carotid artery.

angled lens. In 4 patients $(11.4 \%$; 2 with chordoma, 2 with chondrosarcoma), subtotal removal (> 90\% resection) was performed because the tumor was very fibrous and strongly adherent to critical structures such as cranial nerves or internal carotid arteries. In these cases, radiosurgery was added for the small residual part. Tumor volume was therefore sufficiently reduced in 29 patients (total or subtotal resection, 90.6\%). In 3 patients (9.4\%) chordoma, chondrosarcoma, and clear cell meningioma, one each), tumor components involving the cranial nerves and internal carotid arteries were very hard, resulting in partial mass reduction.

As complications, 6 patients showed mild and transient worsening of cranial nerve symptoms (abducens nerve palsy in 4, ptosis in 1, facial hypesthesia in 1), which gradually improved within a median of 3 months (range, 1 week to 2 years) after surgery. In our cases, intraoperative monitoring of extraocular movement was not useful 
enough to detect those subtle cranial nerve deficits. A patient with recurrent chordoma invading suprasellar regions showed diabetes insipidus and transient amnesia after removal of a tumor component strongly adhering to the ventral hypothalamus, but both complications completely resolved within 6 months.

Postoperative CSF rhinorrhea requiring surgical repair was observed in 1 patient, in whom the chondrosarcoma had invaded into the petrous region involving the internal auditory meatus and jugular bulb. After gross total removal of the tumor, CSF leakage was not apparent during surgery, but suddenly occurred 1 week later. Including this case, pedicled nasoseptal flaps were made for skull base reconstruction in 2 patients.

At the clinical check-up 1 month after surgery, nasal complications (including decreased sense of olfaction or nasal obstruction) were observed in 4 of the 32 patients $(12.5 \%)$. In 1 patient, the tumor was large and extended to the anterior skull base area, and the septal mucosa was dissected close to the upper margin of the anterior skull base area. In this patient, olfactory function showed improvement within 3 months. Another patient who underwent bilateral middle turbinectomy complained of a persistent sense of nasal obstruction at the 1 month check-up, but this resolved within 3 months. Another patient encountered early in our experience underwent bilateral middle turbinectomy at the initial surgical resection, and a pedicled nasal flap was made 1 week later to address postoperative CSF rhinorrhea. Nasal symptoms gradually improved after surgery in this case, but took 6 months to completely resolve. In 1 patient, who had a narrow nasal cavity with markedly congestive nasal mucosa, swelling of nasal mucosa persisted at postoperative endoscopic evaluations 1 week after surgery. At the 1-month check-up, she complained of a persistent sense of nasal obstruction, and endoscopic examination showed synechiae of the nasal mucosa. This persisted at the endoscopic evaluation 3 months postoperatively, considering synechiotomy by the otolaryngologist. As a result, impairment of olfaction or nasal obstruction persisting more than 3 months was found in 1 patient each (3.1\% each).

\section{Discussion}

The most beneficial point of our approach is retaining sufficient approach routes to a wide range of skull base regions while allowing maximal preservation of the nasal mucosa and turbinates. Simultaneously, using the suction tube with irrigating function, without putting an irrigation system on endoscopic lens, we are seldom required to put out the endoscope to clear the distal lens clouded by mucus or blood. Also, instead of holding the endoscope in assistant's hand, we use the robotic endoscope holder to keep the clear stable image of the surgical field, which realizes "endoscopic microsurgery" allowing meticulous surgical procedures.

In skull base surgery through the nostril, the most impeditive anatomic structures on the approach routes are the nasal septum and the middle turbinates. In the current endonasal approach, the mucosa on the sphenoid rostrum and the posterior margin of the nasal septum are removed, and a wide range of ventral skull base regions can be reached. Although this approach method is very simple, in patients with congestive nasal mucosa, adequate surgical approach routes become more and more difficult to maintain over surgical time, and resection of those nasal anatomies become inevitable. ${ }^{11,28)}$ On the other hand, in the transseptal approach, the mucosa is separated from the septum in the unilateral side, and the nasal speculum is inserted beneath the bilateral mucosa on the nasal septum and the posterior vomer. While the mucosa on the sphenoid rostrum can be preserved with a small incision on the septum, in patients who have narrow nasal apertures, speculum blades frequently hamper the approach pathway at the entrance during single-nostril surgery. ${ }^{11)}$ In Asian patients, the size of the nostril is often not large enough to approach from a single nostril and may restrict the surgical performance. ${ }^{13-15)}$ To facilitate the endoscopic skull base surgery with minimum nasal morbidity, we use the nasal specula in an unorthodox fashion.

Use of nasal specula is well known to improve visualization and maintain the surgical pathway while achieving tamponade of the nasal mucosa. ${ }^{28-30)}$ Despite such benefits, it forces the surgeon to choose either a single-nostril approach or a sublabial approach, and to maintain wider surgical pathway, the sublabial approach is sometimes preferred for extended transsphenoidal surgery. ${ }^{27,29)}$ To obtain the benefits of both the modern endoscopic endonasal approach and conventional transsphenoidal surgery using nasal specula, we use shorter specular blades (length of blade: $6 \mathrm{~cm}$ or $7 \mathrm{~cm}$ ) and place them in each nostril. The entireties of both blades are settled in the nasal cavity to retract redundant nasal tissue inside while retaining flexibility of the nasal alae and apex without restricting the approach pathway at the entrance. Using the adjustable speculum specially developed for extended transsphenoidal surgery, ${ }^{27)}$ the opening width and angle of the blades are modified in accordance with individual shape of 
the nasal cavity. Because the specular blades show sufficient fit against the lateral wall of the surgical corridor, we have not found that the working space is noticeably restricted by this equipment. All the anatomical structures impeding a transsphenoidal approach are temporarily dislocated, and the surgical pathway is stably maintained with the nasal speculum from the entrance of the nostril to the deep skull base regions. ${ }^{27,29,30)}$

Since increased risks of sinonasal morbidity and impaired olfaction have been indicated in association with endoscopic skull base surgery, ${ }^{20,22,25,26,31)}$ even in patients with narrow nasal corridors, turbinectomy or harvest of a nasoseptal flap may ideally be performed only after such action really becomes necessary during surgery. From this perspective, our approach offers a "reasonable" risk of nasal morbidity. Even if intraoperative CSF leakage is highly anticipated prior to surgical resection, use of the nasoseptal mucosa can be determined at the last step of the surgical procedure, and preparation of a large pedicled nasoseptal flap in advance is not necessary. As the occasion arises, the pedicled nasoseptal flap is readily tailored according to the size and location of the dural defect for reconstruction. Even in the second surgery, the nasal septum is preserved as in the primary surgery, which can be used for the pedicled flap as necessary. In fact, in most of our patients, the septal mucosa and turbinates could be preserved, and nasal function could be recovered within the early postoperative period. Only 1 patient $(3.1 \%)$ reported persisting olfactory impairment at 3 months after surgery, and the associated risk of moderate-to-severe smell loss is apparently lower than the risks reported in the past literature, $9.5 \%$ to $50 \%$ after the extended endonasal approaches..$^{20,22,26,32)}$ In contrast, postoperative synechia is always anticipated in patients who have severe mucosal swelling with narrow nasal cavity, and special care must be taken to prevent this pathology. In this respect, the role of the otolaryngologist in postoperative patient care will be more important than in the usual endonasal surgery.

In conclusion, our approach allows fine bimanual handling and swift delivery of surgical equipment while offering maximal preservation of the nasal anatomy in patients with prominent nasal mucosa. To successfully reduce nasal complications while improving the efficacy of this surgical method, the otolaryngologist will have a very important role to play in postoperative patient care.

\section{Conflicts of Interest Disclosure}

The authors report no conflict of interest concerning the materials or methods used in this study or the findings specified in this article.

\section{References}

1) Gandhi CD, Christiano LD, Eloy JA, Prestigiacomo CJ, Post KD: The historical evolution of transsphenoidal surgery: facilitation by technological advances. Neurosurg Focus 27: E8, 2009

2) Cappabianca P, Cavallo LM, de Divitiis E: Endoscopic endonasal transsphenoidal surgery. Neurosurgery 55: 933-940; discussion 940-941, 2004

3) Fernandez-Miranda JC, Morera VA, Snyderman CH, Gardner P: Endoscopic endonasal transclival approach to the jugular tubercle. Neurosurgery 71(1 Suppl Operative): 146-158; discussion 158-159, 2012

4) Frank G, Pasquini E, Doglietto F, Mazzatenta D, Sciarretta V, Farneti G, Calbucci F: The endoscopic extended transsphenoidal approach for craniopharyngiomas. Neurosurgery 59(1 Suppl 1): ONS75-ONS83; discussion ONS75-ONS83, 2006

5) Fraser JF, Nyquist GG, Moore N, Anand VK, Schwartz TH: Endoscopic endonasal minimal access approach to the clivus: case series and technical nuances. Neurosurgery 67: ons150-ons158; discussion ons158, 2010

6) Jho HD, Carrau RL: Endoscopic endonasal transsphenoidal surgery: experience with 50 patients. $J$ Neurosurg 87: 44-51, 1997

7) Kassam AB, Gardner PA, Snyderman CH, Carrau RL, Mintz AH, Prevedello DM: Expanded endonasal approach, a fully endoscopic transnasal approach for the resection of midline suprasellar craniopharyngiomas: a new classification based on the infundibulum. J Neurosurg 108: 715-728, 2008

8) Zanation AM, Snyderman CH, Carrau RL, Gardner PA, Prevedello DM, Kassam AB: Endoscopic endonasal surgery for petrous apex lesions. Laryngoscope 119: 19-25, 2009

9) Stippler M, Gardner PA, Snyderman CH, Carrau RL, Prevedello DM, Kassam AB: Endoscopic endonasal approach for clival chordomas. Neurosurgery 64: 268-277; discussion 277-278, 2009

10) Saeki N, Horiguchi K, Murai H, Hasegawa $Y$, Hanazawa T, Okamoto Y: Endoscopic endonasal pituitary and skull base surgery. Neurol Med Chir (Tokyo) 50: 756-764, 2010

11) Shin M, Kondo K, Saito N: Neuroendoscopic transnasal surgery for skull base tumors: basic approaches, avoidance of pitfalls, and recent innovations. Neurol Med Chir (Tokyo) 52: 697-703, 2012

12) Takemura M, Fujimoto $Y$, Kobayashi T, Komori M, Stamm AC, Vellutini E, Mariani P, Kawanishi Y, Shimizu K: A modified combined transseptal/ transnasal binostril approach for pituitary lesions in patients with a narrow nasal space: technical note. Neurol Med Chir (Tokyo) 54: 622-628, 2014

13) Abdelkader M, Leong S, White PS: Aesthetic 
proportions of the nasal aperture in 3 different racial groups of men. Arch Facial Plast Surg 7: 111-113, 2005

14) Morgan NJ, MacGregor FB, Birchall MA, Lund VJ, Sittampalam Y: Racial differences in nasal fossa dimensions determined by acoustic rhinometry. Rhinology 33: 224-228, 1995

15) Pawankar R, Bunnag C, Khaltaev N, Bousquet J: Allergic Rhinitis and Its Impact on Asthma in Asia Pacific and the ARIA Update 2008. World Allergy Organ J 5: S212-S217, 2012

16) Tantilipikorn $P$, Jareoncharsri $P$, Voraprayoon $S$, Bunnag C, Clement PA: Acoustic rhinometry of Asian noses. Am J Rhinol 22: 617-620, 2008

17) Saito K, Toda M, Tomita T, Ogawa K, Yoshida K: Surgical results of an endoscopic endonasal approach for clival chordomas. Acta Neurochir (Wien) 154: 879-886, 2012

18) Shin JH, Kang SG, Kim SW, Hong YK, Jeun SS, Kim EH, Kim SW, Cho JH, Park YJ: Bilateral nasoseptal flaps for endoscopic endonasal transsphenoidal approach. J Craniofac Surg 24: 1569-1572, 2013

19) Taniguchi M, Kohmura E: Endoscopic endonasal removal of laterally extended clival chordoma using side-viewing scopes. Acta Neurochir (Wien) 154: 627-632, 2012

20) Alobid I, Enseñat J, Mariño-Sánchez F, de Notaris M, Centellas S, Mullol J, Bernal-Sprekelsen M: Impairment of olfaction and mucociliary clearance after expanded endonasal approach using vascularized septal flap reconstruction for skull base tumors. Neurosurgery 72: 540-546, 2013

21) Chhabra N, Houser SM: The diagnosis and management of empty nose syndrome. Otolaryngol Clin North Am 42: 311-330, ix, 2009

22) de Almeida JR, Snyderman CH, Gardner PA, Carrau RL, Vescan AD: Nasal morbidity following endoscopic skull base surgery: a prospective cohort study. Head Neck 33: 547-551, 2011

23) Eloy JA, Patel AA, Shukla PA, Choudhry OJ, Liu JK: Early harvesting of the vascularized pedicled nasoseptal flap during endoscopic skull base surgery. Am J Otolaryngol 34: 188-194, 2013

24) El-Sayed IH, Roediger FC, Goldberg AN, Parsa AT,
McDermott MW: Endoscopic reconstruction of skull base defects with the nasal septal flap. Skull Base 18: 385-394, 2008

25) Georgalas C, Badloe R, van Furth W, Reinartz S, Fokkens WJ: Quality of life in extended endonasal approaches for skull base tumours. Rhinology 50: 255-261, 2012

26) Pant H, Bhatki AM, Snyderman CH, Vescan AD, Carrau RL, Gardner P, Prevedello D, Kassam AB: Quality of life following endonasal skull base surgery. Skull Base 20: 35-40, 2010

27) Kitano M, Taneda M: An adjustable nasal speculum for the extended transsphenoidal approach. Technical note. J Neurosurg 106: 932-933, 2007

28) Zada G, Governale LS, Laws ER: Intraoperative conversion from endoscopic to microscopic approach for the management of sellar pathology: incidence and rationale in a contemporary series. World Neurosurg 73: 334-337, 2010

29) Al-Mefty O, Kadri PA, Hasan DM, Isolan GR, Pravdenkova S: Anterior clivectomy: surgical technique and clinical applications. J Neurosurg 109: 783-793, 2008

30) Kitano M, Taneda M, Shimono T, Nakao Y: Extended transsphenoidal approach for surgical management of pituitary adenomas invading the cavernous sinus. J Neurosurg 108: 26-36, 2008

31) Kimple AJ, Leight WD, Wheless SA, Zanation AM: Reducing nasal morbidity after skull base reconstruction with the nasoseptal flap: free middle turbinate mucosal grafts. Laryngoscope 122: 1920-1924, 2012

32) Gallagher MJ, Durnford AJ, Wahab SS, Nair S, Rokade A, Mathad N: Patient-reported nasal morbidity following endoscopic endonasal skull base surgery. Br J Neurosurg 28: 622-625, 2014

Address reprint requests to: Masahiro Shin, $\mathrm{MD}, \mathrm{PhD}$, Department of Neurosurgery, The University of Tokyo Hospital, 7-3-1 Hongo, Bunkyo-ku, Tokyo 133-8655, Japan. e-mail: SHIN-NSU@h.u-tokyo.ac.jp 